\title{
FINE STRUCTURE EVENTS IN MICROWAVE EMISSION DURING SOLAR MINIMUM
}

\author{
Chengming Tan \\ National Astronomical Observatories, Chinese Academy of Sciences, \\ Beijing, China, tanchm@nao.cas.cn \\ University of Chinese Academy of Sciences, \\ Beijing,China,tanchm@nao.cas.cn \\ Baolin Tan \\ National Astronomical Observatories, Chinese Academy of Sciences, \\ Beijing,China,bltan@nao.cas.cn \\ University of Chinese Academy of Sciences, \\ Beijing,China,bltan@nao.cas.cn

\section{Yihua Yan} \\ National Astronomical Observatories, Chinese Academy of Sciences, \\ Beijing,China,yyh@nao.cas.cn \\ University of Chinese Academy of Sciences, \\ Beijing,China,yyh@nao.cas.cn
}

\author{
Wei Wang \\ National Astronomical Observatories, Chinese Academy of Sciences, \\ Beijing, China,wwang@nao.cas.cn \\ Linjie Chen \\ National Astronomical Observatories, Chinese Academy of Sciences, \\ Beijing, China, ljchen@nao.cas.cn \\ Fei Liu \\ National Astronomical Observatories, Chinese Academy of Sciences, \\ Beijing, China,feiliu@nao.cas.cn \\ Yujiang Dou \\ Soochow University, \\ Suzhou,China,douyj@suda.edu.cn
}

Abstract. The solar minimum is a period with a relatively smaller number of sunspots and solar eruptions, and has been less studied before. Since the radio signal rapidly responds to the change of solar plasma and magnetic field, we perform a comprehensive analysis of high resolution spectrum data from SBRS and MUSER: 1) a search for solar radio bursts of different kinds in recent solar minima (2007-2009 and 2016-2018); 2) an analysis of several typical radio burst events, negative and positive drifting bursts, for example the November 22, 2015 and August 29, 2016 events; superfine spectral structure events with mini-flares and even without sunspots, for example the March 28, 2008 and July 04, 2017 events.
These results show that there were many radio bursts with a fine structure during solar minima. These events occurred not only in powerful flares, but also in faint flares (class C and B by GOES) or even without flares, but in regions related to weak brightenings or ejecta. We assume that the weak solar radio bursts observed by telescopes with high sensitivity and low interference will help us to understand the basic physical characteristics of small-scale solar eruptions.

Keywords: solar minimum, flare, radio burst, spectrum, fine structure.

\section{INTRODUCTION}

In recent years, the study of solar minima has mainly focused on solar activities [Lingri et al., 2016], magnetic field characteristics [de Toma et al., 2000], etc. With a series of high performance solar instruments developed, it has been found that there are many small-scale eruptions in solar minima. The solar dynamo shows that features of magnetic activity at solar minimum are likely to determine the intensity and duration of the next solar cycle [Tan, 2019]. The study of the radio emission at solar minimum mainly focuses on characteristics of radiation of the quiet Sun, paying little attention to nonthermal explosion phenomena. A reason is that there are few active regions, weak magnetic fields, and radio bursts during solar minima. Tan et al. [2013] have analyzed all observations made with the China Solar Broadband Radio Spectrometer (SBRS) [Fu et al., 1995, 2004; Ji et al., 2000] from 1997 to 2011. It has been found that only flares above M4.0 class are accompanied by microwave bursts by $100 \%$. Some low-class flares occur without microwave bursts. There are few radio bursts recorded in 2008-2009. On the other hand, the instrument is sensitive to electromagnetic interference. In the data analysis, it is difficult to distinguish weak bursts from interference. Recently, we have tried to improve data processing methods and software [Chen et al., 2016], gained more experience in identifying weak bursts from interference signals. When analyzing high resolution observation data during the solar minima, more bursts have been found. It has been established that radio bursts and superfine spectral structures still occur in a mini-flare or weak magnetic field region. There are many studies [Altyntsev, 2007, 2008; Chernov, 2006, 2011] of the radio fine spectral structures which happen during strong solar flares. Zhdanov and Zandanov [2015] have found that fine structures are detected both during solar flares accompanied by microwave broadband emission and during weak solar flares when the microwave broadband emission is absent. Thus, we try to study radio fine spectral structure events during solar minima because there are almost no works on this case for now. As the preliminary results we present four events here.

\section{INSTRUMENT AND OBSERVATION}

We study the radio spectrum observed by SBRS during the solar minima of 2007-2009 and 2016-2018. The SBRS1 spectrometer has been upgraded three times [Tan et al., 2015]. The newest receiver was developed by Soochow University and has been working since 
2013. Now SBRS1 parameters are as follow:

- Antenna diameter: $7.4 \mathrm{~m}$;

- Frequency band: 1.10-2.10 GHz;

- Frequency resolution: $2.78 \mathrm{MHz}$;

- Frequency channels: 360;

- Time cadence: $5 \mathrm{~ms}$;

- Accuracy of polarization: $<10 \%$.

Hundreds of solar radio bursts were identified during the 2007-2009 and 2016-2018 solar minima; some of them were accompanied by very weak flares (miniflares). As a comparison, we study the radio spectrum observed by Mingantu Spectral Radioheliograph (MUSER) [Yan et al., 2016]. MUSER is a new aperture synthesis solar radio telescope array, which can observe the full Sun in centimeter-decimeter wavelengths with high resolutions and dual polarization. The maximum baseline is $3 \mathrm{~km}$, which gives the maximum spatial resolutions from $63^{\prime \prime}$ to $1.7^{\prime \prime}$ at different frequencies. MUSER is composed of two sub-arrays: MUSER-I and MUSER-II with frequency ranges $0.4-2.0 \mathrm{GHz}$ and 2.0 $15.0 \mathrm{GHz}$ respectively. The whole array is composed of 100 antennas distributed in three spiral arms with the longest baseline of $3.0 \mathrm{~km}$. The dynamic range is designed to be $25 \mathrm{~dB}$, the temporal resolution is $25 \mathrm{~ms}$, and the frequency resolution is $25 \mathrm{MHz}$. The measurement accuracy of the dual circular polarization is about $10 \%$. MUSER-I has been working since 2014; and MUSER-II, since the summer of 2016. In this paper, we only adopted the spectrograms obtained by MUSER-I.

The GOES soft X-ray flux [Bornmann et al., 1996], SDO AIA [Pesnell et al., 2012] image, and RHESSI [Lin et al., 2002] hard X-ray observations are also used here for analysis.

\section{DATA AND EVENT ANALYSIS}

Few radio burst events have previously been recorded from SBRS data during solar minima. The bursts were weak and had short duration. Due to the development of calibration [Tan et al., 2015], interference cleaning, and image recognition [Chen et al., 2016], more and more weak solar radio bursts have been observed and identified from observations with new telescopes. Here we illustrate four examples. Among them are two events with negative and positive drift velocities, which are accompanied by very weak GOES soft $\mathrm{X}$-ray flares. The third event is type III bursts with a very weak flare identified, and the fourth event is superfine structure bursts with no sunspots reported from the full-Sun observations from March 3, 2017 to July 4, 2017.

The November 22, 2015 event is shown in Figure 1. The radio fine structure occurred between 04:51:27 and 04:51:41 UT. First, groups of narrowband type III bursts drifted slowly from high to low frequencies, and then they drifted slowly and reversely to high frequencies. It is also very interesting that most of individual type III bursts also drifted in the same way as the groups, i.e. they first drifted from high to low frequencies and then reversely to high frequencies. These groups of type III bursts appeared just at the beginning of a small knot of flare $(\mathrm{C} 1.2$ class indicated by an arrow on the top panel of Figure 1). Thus we may imagine that electron beams accelerated and traveled along the magnetic loop top, but failed to escape to the upper corona. Then they went downward to another loop foot.

The August 29, 2016 event is shown in Figure 2. The radio fine structure occurred between 03:44:40 and 03:47:00 UT, with weak right circular polarization. Groups of narrowband type III bursts first drifted slowly from low to high frequencies, then drifted up and down, and afterwards drifted slowly upward to high frequencies. It is also very interesting that most of the individual type III bursts also drifted in the same way as the groups, i.e. they first drifted from low to high frequencies, then drifted upward to low frequencies. These groups of type III bursts occurred just at the maximum of a moderate flare ( $\mathrm{C} 2.2$ class indicated by an arrow on the top panel of Figure 2). Thus, we may assume that electron beams were accelerated within the magnetic reconnection region, and traveled up and down along the magnetic loop; some of them managed to escape to the upper corona.

The March 28, 2008 event is shown in Figure 3. The radio fine structure occurred between 00:14:30 and 00:15:05 UT. It is a group of type III bursts. Each individual group of type III bursts drifted fast downward to high frequencies. This group of type III bursts has no corresponding flare identified nearby. The upper two panels of Figure 3 indicate that there is no SXR flare but a weak HXR flare at 6-12 keV observed by RHESSI approximately at the same time as the type III bursts were recorded. The two bottom panels are the EIT $195 \AA$ image after running differentiation, superposed onto the 6-12 keV RHESSI image between 01:11:22 and 01:17:22 UT. There are no RHESSI images for 00:00-00:30 UT.

The July 04, 2017 event is shown in Figure 4. The radio fine structure occurred between 01:07:46.14 and 01:07:46.55 UT, for less than 500 milliseconds. Each individual burst lasted for $<20 \mathrm{~ms}$. It is a little difficult to identify if there is a drift or not for each individual group of type III bursts since its duration is approximately equal to the time resolution. The first panel of Figure 4 shows the radio fine structure (time is indicated by an arrow) is just before the maximum of a small flare (B2.0 class). There are no sunspots or active regions reported for this group of radio fine structures. The AIA $171 \AA$ full-disk solar image exhibits no remarkable flares or ejecta within 01:00-01:10 UT. The AIA 171 $\AA$ west limb images (bottom four panels) display weak brightening near the limb. There are no MUSER observations for the time of this radio fine structure.

\section{RESULTS AND DISCUSSION}

In this work, we only report that there were some radio bursts accompanied by weak solar flares (miniflares) during solar minima. From the four events analyzed, several results can be summarized as follows:

1. During solar minima, the radio burst spectrum also exhibited many fine structures, including groups of 

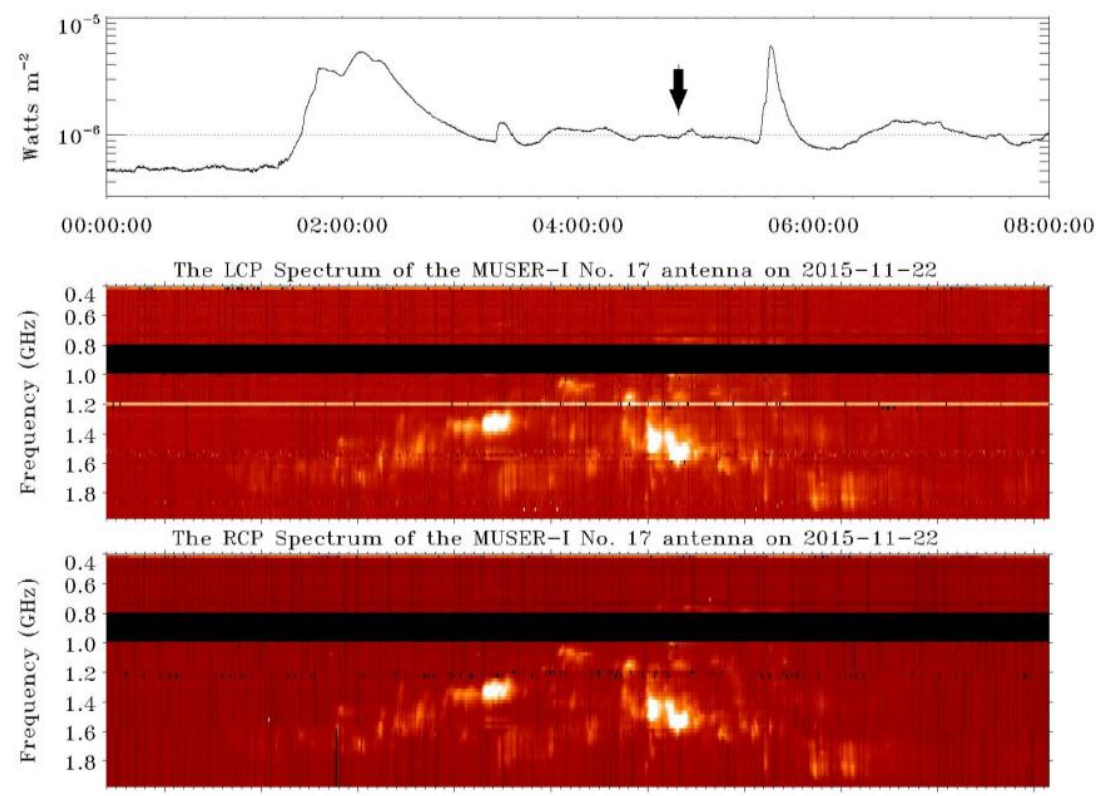
$04: 51: 26$
04:51:30
$04: 51: 34$
$04: 51: 38$
$04: 51: 42$
טт

Figure 1. The November 22, 2015 event. The top panel is the GOES soft X-ray at 1.0-8.0 $\AA$. The black arrow indicates the time of corresponding radio fine structure spectrum shown on two bottom panels. The radio spectrum is from MUSER observation
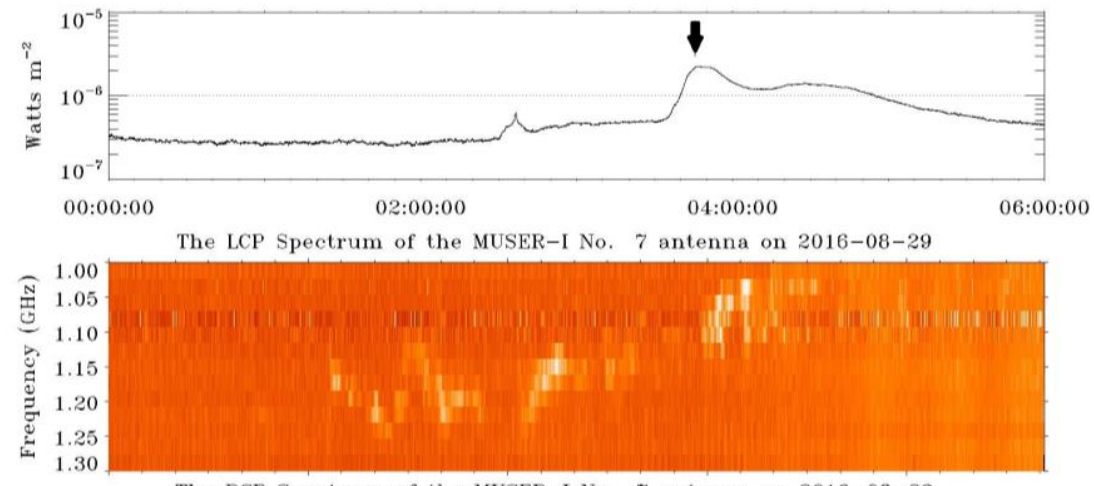

The RCP Spectrum of the MUSER-I No. 7 antenna on 2016-08-29

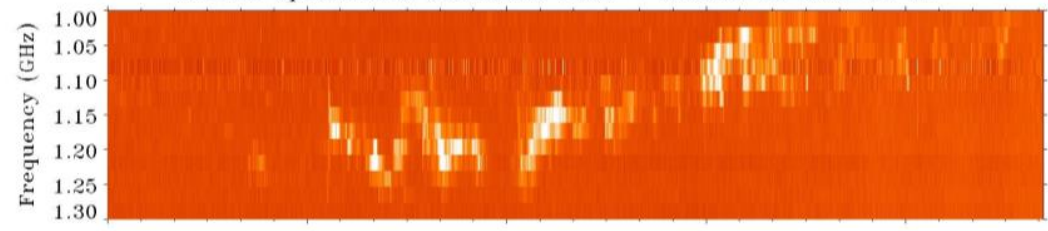
$03: 44: 30$
03:45:00
03:45:30
03:46:00
$03: 46: 30$

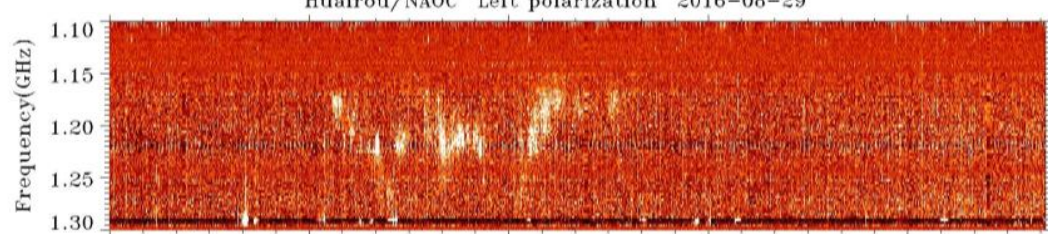

Right polarization

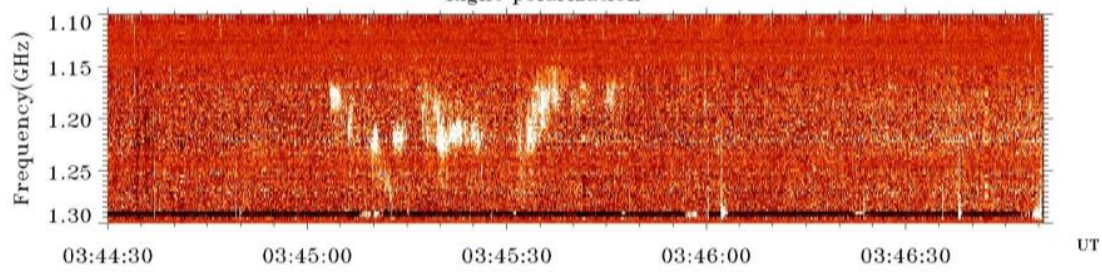

Figure 2. The August 29, 2016 event. The top panel is the GOES soft X-ray at 1.0-8.0 $\AA$. The black arrow indicates the time of corresponding radio fine structure spectrum shown on four bottom panels. Two panels of radio spectrum are from MUSER observation. Another two panels of radio spectrum are from SBRS1 observation 

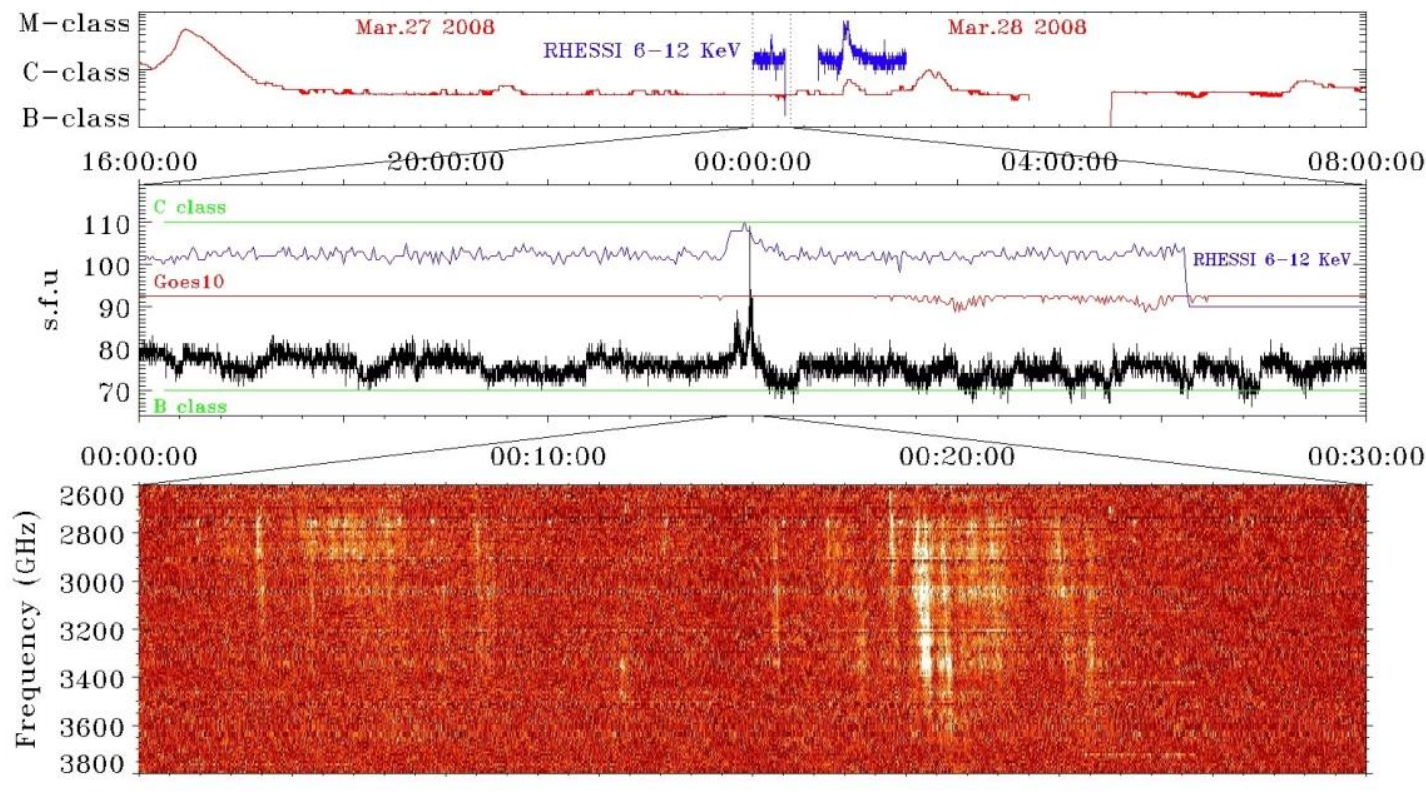
$00: 14: 30$
00:14:40
$00: 14: 50$
00:15:00
00:15:10
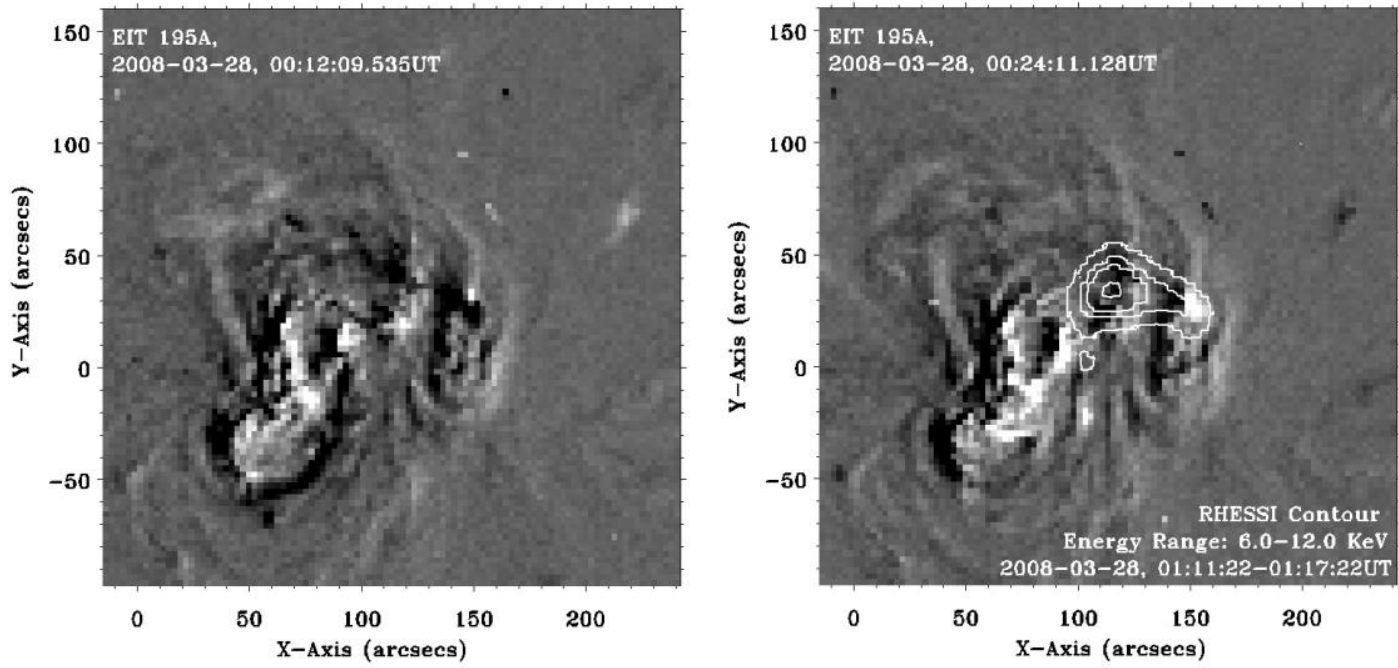

Figure 3. The March 28, 2008 event. The first panel is the GOES soft X-ray at 1.0-8.0 $\AA$. The second panel plots the profile of GOES soft X-ray and radio flux observed by SBRS at $2940 \mathrm{MHz}$. The RHESSI 6-12 keV profile is also plotted on both of the panels. On the third panel is the radio fine structure spectrum observed by SBRS at the $2600-3800 \mathrm{MHz}$ band. Two bottom panels present the EIT $195 \AA$ image after running differentiation, superposed onto the RHESSI image

drifting type III bursts, etc. They occurred not only during the main solar flare, but also during small flares, mini-flares, and even without obvious flares and sunspots. Some radio fine structures occurred without obvious flares or sunspots, but still with weak EUV brightening or ejecta, or with HXR microflare.

2. The first two radio fine structure events are groups of type III bursts drifting up and down globally, while the third one are groups of type III bursts drifting down globally. For all the three events, the individual type III burst drifted in the same way as that drifting globally. The fourth one is a superfine structure event with short group duration of $<500 \mathrm{~ms}$ and short individual duration of $<20 \mathrm{~ms}$. Some structures have unusual duration of $<5 \mathrm{~ms}$ and strong intensity. It is difficult to identify if the individual structure drifted or not.

The globally drifting type III bursts might have similar physical evolvement as the drifting pulsation struc- tures (DPSs) which usually happened in the initial phase of a flare. DPSs [Karlicky, 2004; Tan et al., 2008] usually are groups of up and down drifting bursts. Karlicky [2004] has explained the DPS map of the evolution of the primary and secondary plasmoids formed due to tearing and coalescence instabilities in the current sheet during the reconnection process. Melnikov et al. [2002, 2005] suggested that the flare loop is filled with dense plasma with the density number $n_{0} \approx 10^{11} \mathrm{~cm}^{-3}$ and that accelerated electrons are concentrated in the upper part of the flare loop. Zhdanov and Zandanov [2015] have reported that radio fine structures can be detected during weak solar flares when the microwave broadband emission is absent. Nakariakov et al. [2018] have reported on radio quasi-periodic pulsations (QPP) in a B2-microflare. QPP are likely to be caused by the superposition of the signals generated at local electron plasma frequencies by the interaction of nonthermal 

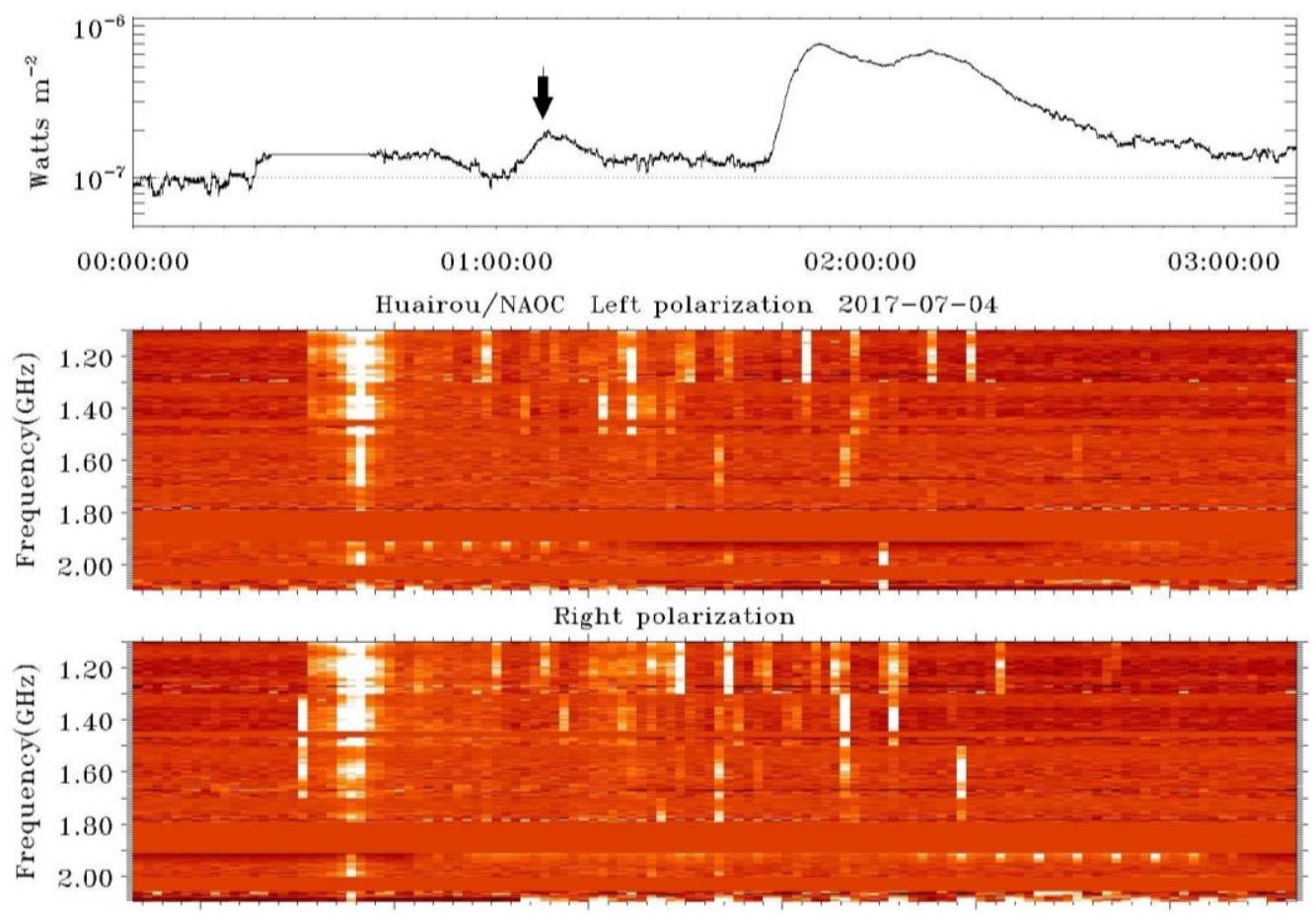

01:07:46.10

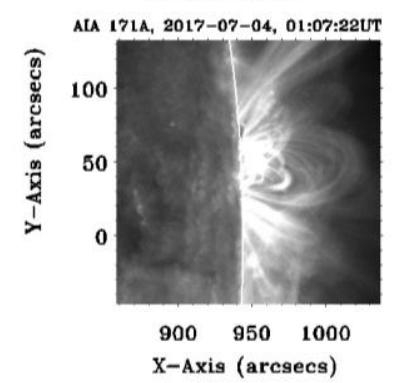

$01: 07: 46.30$

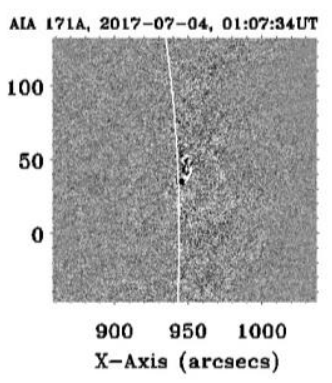

01:07:46.50 UT

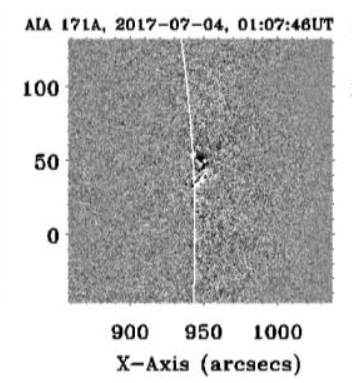

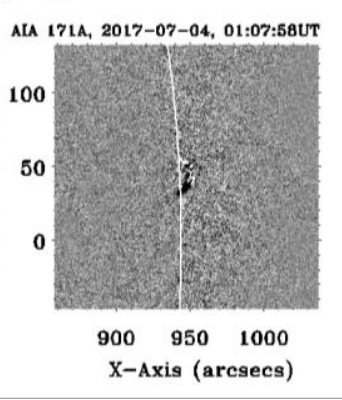

Figure 4. The July 04, 2017 event. The top panel is the GOES soft X-ray at 1.0-8.0 A. The black arrow indicates the time of corresponding radio fine structure spectrum observed by SBRS at 1100-1900 MHz band, shown on the second panel. The four bottom panels are SDO AIA $171 \AA$ images after running differentiation

electrons with plasma at footpoints. All these help us to understand that radio fine structures can still occur in a weak flare during solar minimum as long as an abundance of electrons are accelerated during the reconnection along the loop with dense plasma. Thus, the fine structure can still be observed with a high-sensitivity and resolution telescope.

Our future work will compare more events with other observations at a similar frequency band, for example SSRT [Smolkov et al., 1986; Grechnev et al., 2003] and YNRS [Gao et al., 2014], and study the flare loop with radio image observations from MUSER and NORH [Nakajima, 1994]. We can expect that the weak radio bursts observed with the high-sensitivity lowinterference telescope will help us to understand physical characteristics of small-scale eruptions and nanoflares, and will further expand our understanding of solar radio emission and solar magnetic field.

This work is supported by NSFC grants Nos. 11373039, 11433006, 11573039, 11661161015, 11790301, and 11790305. We thank NGDC, Solarmonitor, SWPC, and Space Weather Prediction Center for online data.

\section{REFERENCES}

Altyntsev A.T., Grechnev V.V., Meshalkina N.S., Yan Y. Microwave type III-like bursts as possible signatures of magnetic reconnection. Solar Phys. 2007, vol. 242, iss. 1-2, pp. 111123. DOI: $10.1007 / \mathrm{s} 11207-007-0207-9$.

Altyntsev A.T., Fleishman G.D., Huang G.-L., Melnikov V.F. A broadband microwave burst produced by electron beams. Astrophys. J. 2008, vol. 677, iss. 2, pp. 1367-1377. DOI: 10.1086/ 528841.

Bornmann P.L., Speich D., Hirman J., Matheson L., Grubb R., Garcia H., Viereck R. GOES X-ray sensor and its use in predicting solar-terrestrial disturbances. Proc. SPIE. 1996, vol. 2812, pp. 291-298. DOI: 10.1117/12.254076.

Chen Zhuo, Ma Lin, Xu Long, Yan Y. Imaging and representation learning of solar radio spectrums for classification. Multimedia Tools and Applications. 2016, vol. 75, no. 5, pp. 28592875. DOI: $10.1007 / \mathrm{s} 11042-015-2528-2$.

Chernov G.P. Solar radio bursts with drifting stripes in emission and absorption. Space Sci. Rev. 2006, vol. 127, iss. 1-4, pp. 195-326. DOI: 10.1007/s11214-006-9141-7.

Chernov G.P. Fine structure of solar radio bursts. Astrophysics and Space Science Library. Springer-Verlag. Berlin Heidelberg, 2011, vol. 375, 375 p.

de Toma G., White O.R., Harvey K.L. Picture of solar 
minimum and the onset of solar cycle 23. I. Global magnetic field evolution. Astrophys. J. 2000, vol. 529, iss. 2, pp. 11011114. DOI: $10.1086 / 308299$.

Fu Q.J., Qin Z.H., Ji H.R., Pei L. Broadband spectrometer for decimeter and microwave radio bursts. Solar Phys. 1995, vol. 160, iss. 1, pp. 97-103. DOI: 10.1007/BF00679098.

Fu Q., Ji H., Qin Z., Xu Z., Xia Z., Wu H., et al. A New Solar Broadband Radio Spectrometer (SBRS) in China. Solar Phys. 2004, vol. 222, iss. 1, pp. 167-173. DOI: 10.1023/B:SOLA 0000036876.14446.dd.

Gao G., Wang M., Dong, L., Wu N., Lin J. Decimetric and metric digital solar radio spectrometers of the Yunnan Astronomical Observatories and the first-light results. New Astronomy 2014, vol. 30, pp. 68-78. DOI: 10.1016/j.newast.2014.01.008.

Grechnev V.V., Lesovoi S.V., Smolkov G.Ya., Krissinel B.B., Zandanov V.G., Altyntsev A.T., Kardapolova N.N., Sergeev R.Y., Uralov A.M., Maksimov V.P., Lubyshev B.I. The Siberian Solar Radio Telescope: the current state of the instrument, observations, and data. Solar Phys. 2003, vol. 216 , iss. 1, pp. 239-272. DOI: 10.1023/A:1026153410061.

Ji Hui-rong, Fu Qi-jun, Liu Yu-ying, Cheng Cong-ling, Chen Zhi-jun, Lao De-bang, et al. A radio spectrometer at 2.6-3.8 GHz. Chinese Astron. Astrophys. 2000, vol. 24, iss. 3 , pp. 387-393. DOI: 10.1016/S0275-1062(00)00068-0.

Karlicky M. Series of high-frequency slowly drifting structures mapping the flare magnetic field reconnection. Astron. Astrophys. 2004, vol. 417, p. 325-332. DOI: 10.1051/0004 6361:20034249.

Lin R.P., Dennis B.R., Hurford G.J., Smith D.M., Zehnder A., Harvey P.R., et al. The Reuven Ramaty High-Energy Solar Spectroscopic Imager (RHESSI). Solar Phys. 2002, vol. 210, iss. 1, pp. 3-32. DOI: 10.1023/A:1022428818870.

Lingri D., Mavromichalaki H., Belov A., Eroshenko E., Yanke V., Abunin A., Abunina M. Solar activity parameters and associated Forbush decreases during the minimum between cycles 23 and 24 and the ascending phase of cycle 24. Solar Phys. 2016, vol. 291, iss. 3, pp.1025-1041. DOI: 10.1007/s11207-016-0863-8.

Melnikov V.F., Shibasaki K., Reznikova V.E. Loop-top nonthermal microwave source in extended solar flaring loops. Astrophys. J. 2002, vol. 580, iss. 2, pp. L185-L188. DOI: $10.1086 / 345587$.

Melnikov V.F., Reznikova V.E., Shibasaki K., Nakariakov V.M. Spatially resolved microwave pulsations of a flare loop. Astron. Astrohys. 2005, vol. 439, iss. 2, pp. 727-736. DOI: 10.1051/0004-6361:20052774.

Nakajima H., Nishio M., Enome S., Shibasaki K., Takano T., Hanaoka Y., et al. The Nobeyama Radiohel' graph. Proc. IEEEP. vol. 82, no. 5, pp. 705-713.

Nakariakov V.M., Anfinogentov S., Storozhenko A.A., Kurochkin E.A., Bogod V.M., Sharykin, I.N., Kaltman T.I. Quasi-periodic pulsations in a solar microflare. Astrophys. J. vol. 859 , iss. 2 , article id. 154,8 p. DOI: $10.3847 / 1538$ 4357/aabfb9.

Pesnell W.D., Thompson B.J., Chamberlin P.C. The Solar Dynamics Observatory (SDO). Solar Phys. 2012, vol. 275 , iss. 1-2, pp. 3-15. DOI: 10.1007/s11207-011-9841-3.

Smolkov G.Ya., Pistolkors A.A., Treskov T.A., Krissinel B.B., Putilov V.A., Potapov N.N. The Siberian Solar Radio-Telescope - parameters and principle of operation, objectives and results of first observations of spatio-temporal properties of development of active regions and flares. Astrophys. Space Sci. 1986, vol. 119, iss. 1, pp. 1-4. DOI: 10.1007/BF00648801.

Tan B.L. The characteristics of valley phase as predictor of the forthcoming solar cycle. Adv. Space Res. 2019, vol. 63, iss. 1, pp. 617-625. DOI: 10.1016/j.asr.2018.08.004.

Tan C., Yan Y.H., Liu Y.Y., Jing H.R. Statistical study of radio drifting pulsation structures with associated CMEs and other observations. Adv. Space Res. 2008, 41, no. 6, pp. 969-975.
DOI: 10.1016/j.asr.2007.04.085

Tan C., Tan B.L., Yan Y.H., Liu Y. Microwave observations of the Chinese Solar Broadband Radio Spectrometer at Huairou. Solar and Astrophysical Dynamos and Magnetic Activity: Proc. of the International Astronomical Union, IAU Symposium. 2013, vol. 294, pp. 499-500. DOI: 10.1017/S1743921313003037.

Tan C., Yan Y., Tan B., Fu Q., Liu Y., Xu G. Study of calibration of solar radio spectrometers and the quiet-Sun radio emission. Astrophys. J. 2015, vol. 808, iss. 1, article id. 61, 14 p.

Yan Y., Chen L., Yu S. First radio burst imaging observation from Mingantu Ultrawide Spectral Radioheliograph. Solar and Stellar Flares and their Effects on Planets: Proc. of the International Astronomical Union, IAU Symposium. 2016, vol. 320, pp. 427-435. DOI: 10.1017/S174392131600051X.

Zhdanov D.A., Zandanov V.G. Observations of Microwave fine structures by the Badary Broadband Microwave Spectropolarimeter and the Siberian Solar Radio Telescope. Solar Phys. 2015, vol. 290, iss. 1, pp. 287-294. DOI: 10.1007/s11207-014-0553-3.

\section{Color Figures are available in the article electronic version.}

How to cite this article

Chengming Tan, Baolin Tan, Yihua Yan, Wei Wang, Linjie Chen, Fei Liu, Yujiang Dou. Fine structure events in microwave emission during solar minimum. Solar-Terrestrial Physics. 2019. Vol. 5. Iss. 2. P. 3-8. DOI: $10.12737 /$ stp-51201901. 